\title{
Reply to Wray
}

\section{Eugenio Petrovich ${ }^{1}$}

\begin{abstract}
In this letter, I reply to two insightful remarks made by Wray on a study I recently published on Scientometrics. First, I clarify the method I used to assess the transition of Analytic Philosophy to a normal science phase, explaining in more details the epistemological role that different types of citations play. Second, I address the topic of the relationship between the normal science and the aging of the literature. I argue that more research is needed to understand the epistemological meaning of aging.
\end{abstract}

Keywords: Normal science, Aging of the literature, Kuhn, Analytic Philosophy, Price Index

In his note, Wray takes issue with the method I used in a recent paper to measure the "normalization" of Analytic Philosophy (Petrovich, 2018). By normalization, I mean the approaching of Analytic Philosophy to a Kuhnian normal science style of intellectual production. I would like first to thank Wray for his remarks because they allow me to better clarify the relationship between the different epistemological functions of citations (especially the Supporting citations) and the Kuhnian notion of “normal science". Moreover, Wray's note allows me to deal with an important topic I did not address in my study, namely the relationship between the normal science and the aging of the literature. In this reply, I will firstly focus on the first point and then address this subject.

\section{Normal science and the epistemological functions of citations}

Concerning the first point, let me first sum up my use of the Kuhnian theory of normal science. In my study, I focused on a particular aspect of this theory, namely the fact that only in normal science periods, knowledge progresses in a cumulative nature (see the third paragraph of the article). Thus, the key concept of my study is knowledge accumulation. We may define it as the process in which new knowledge claims are produced and put in relation, in different ways, with the previous knowledge claims. Very roughly,

\footnotetext{
${ }^{1}$ eugenio.petrovich@,unimi.it, Department of Philosophy, University of Milan, Via Festa del Perdono 7, 20122 Milan, Italy
} 
knowledge claims can conflict to prior claims, or they can be added cumulatively to the previous ones. The first scenario is typical of what Kuhn calls the "pre-paradigmatic" science, where knowledge is continuously contested, and each scientist has to rebuild the field from scratch every time. The second scenario is what we expect in normal science, and it is the reason why Kuhn defines normal science as progressive.

As I argue in the fourth paragraph of my article, I believe that citations are particularly relevant to investigate the process of knowledge accumulation because they represent the explicit links that knowledge producers (i.e., authors) establish between their new contributions (i.e., their publication) and the existing collection of pieces of knowledge (i.e., the literature). The kind of link that the authors establish indicates whether the process is cumulative (as in normal science) or non-cumulative (as in preparadigmatic science). Indeed, three categories of the classificatory scheme of my study were designed to capture the epistemological relationships between the citing and the cited text. Specifically, State of the art citations were designed to pick up the presence of a knowledge base (a shared literature), Supporting citations to capture constructive operations in which new knowledge is added, and Critical citations destructive operations in which the citing text undermines a previous knowledge claim.

Now, in normal science, we would expect, respectively, a high rate of Supporting citations (the citations that are directly connected with the progress of knowledge), a high rate of State of the art citations (indicating the presence of a shared knowledge base), and a low rate of Critical citations (meaning that knowledge is not contested). ${ }^{2}$ Therefore, my assessment of the "normalization" of Analytic Philosophy depended on the assessment of the rates of all the three types of citations, not only of Supporting citations. In particular, I think that the State of the art citations are crucial in assessing the normalization of a field because they indicate that the context of the research is clear. Indeed, only in the normal science, there can be a "state of the art". In pre-paradigmatic science each author begins from zero, so to say. State of the art citations attests that analytic philosophers share a common idea of the structure of the field because they use them to locate their contribution into a precise stream of research. I think this is a significant step towards the normalization of Analytic Philosophy since it shows that at least the shape of the field is no more contested. Such a result may appear trivial in the case of the sciences but in philosophy is a remarkable achievement since the taxonomy of philosophy is indeed a philosophical problem (Rescher, 2014). Thus, I think that it is plausible to say that the increase of State of the art citations is a sign of the normalization process. From this point of view, I do think that Analytic Philosophy has come close to normal science.

\footnotetext{
${ }^{2}$ Indeed, this is the picture that emerges from previous citation context studies of the sciences in their normal phase (see the studies cited in my article).
} 
However, as I argue in the Discussion, the unstable trend of Supporting citation invites some caution before assimilating straightforwardly Analytic Philosophy to the normal science. The lack of an increasing trend in this type of citations indicates that, within each of the sub-areas of Analytic Philosophy, knowledge is still contested. No strong paradigm, in the Kuhnian sense of the world, has emerged. In order to explain this paradoxical situation, I advanced the hypothesis that Analytic Philosophy has fragmented into sub-disciplinary areas, producing at the same time, in its practitioners, the awareness of a clear sub-disciplinary structure (i.e., the idea that Analytic Philosophy is subdivided into metaphysics, philosophy of mind, philosophy of language, and so on). I described this process as the emergence of a "soft paradigm". The soft paradigm regards the knowledge of the structure of the field. It assures that this structure is not contested. On the other hand, as I said before, within each sub-disciplinary area, knowledge is still unstable.

In sum, I do think that Analytic Philosophy has undergone a process of normalization. Yet, it has become a suigeneris normal science, denoted by a soft paradigm and a fragmented structure. Hence, I do believe that the Kuhnian model offers precious insights also on non-strictly-scientific fields. However, it cannot be mechanically applied to them, but it has to be developed and adapted.

\section{Normal science and the aging of the literature}

In the second part of his note, Wray proposes some insight into how we might better measure normal science in future studies. He suggests that «what we should expect if a field is advancing into a normal science state is that more recently published papers are citing more recently published literature». This idea seems plausible and, as Wray recalls, there is a stream of scientometric research that is devoted indeed to studying the phenomena of aging and obsolescence in the scientific literature. Moreover, the famous Price Index was based on a similar insight. The Price Index is the percentage of references, for a given year, to material that is $\mathrm{X}$ years old or younger (Price used a threshold of 5 years, but other thresholds can be used, see Glänzel \& Schoepflin, 1995). Price showed that the sciences, the social sciences, and the humanities could be distinguished based on the Price Index. Specifically, the sciences present high values of the Price Index, the social science an intermediate value, and the humanities a low value (Price, 1986).

Thus, it seems plausible to claim that there is a correlation between the shrinking age of the literature (measured either by the Price Index ${ }^{3}$ or by the average age of the cited literature) and the transition to the normal science phase. ${ }^{4}$ However, a recent study of the evolution of the Price Index over time has shown an unexpected result: «contrary to a widely held belief, science as a whole - and especially NSE

\footnotetext{
${ }^{3}$ Note that the Price Index is only slightly affected by the outliers of the cited references list (i.e., the youngest and the oldest item), since it is a percentage and not an average. See (Wray, 2016).

${ }^{4}$ Indeed, before turning to the citation context analysis, I attempted to use the age of the literature as indicator of the normalization process of Analytic Philosophy.
} 
[natural sciences and engineering] - relies on an increasingly older body of literature» (Larivière, Archambault, \& Gingras, 2008, p. 296). Larivière et al. demonstrate that the Price Index of both medical sciences and natural sciences and engineering followed a decreasing trend since 1955 (Larivière et al., 2008, fig. 4), showing that «in contrast to a widely held belief, scientific literature is not becoming obsolete more rapidly than it used to; in fact, quite the opposite is observed» (295). I think that these findings urge some caution before using the shrinking age as a proxy for the passage to normal science state since paradigmatic cases of normal sciences do not present the decreasing pattern we would expect. ${ }^{5}$

Furthermore, the trend of the Price Index of Analytic Philosophy does not increase over time (see Fig. 1 and 2), neither using a 5- nor a 10-year threshold.

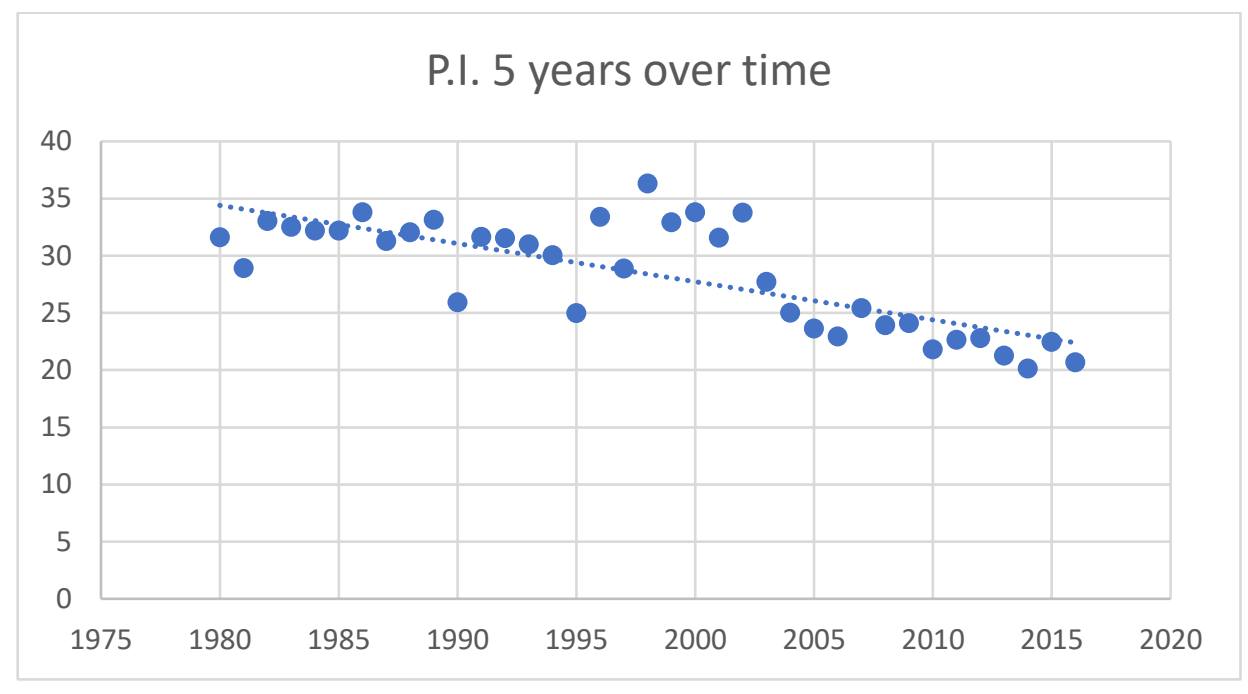

Figure 1. Price Index of Top Five Analytic Philosophy journals (5 years)

${ }^{5}$ Furthermore, Egghe has showed that both the decrease of the mean and median age of the references, as well as the decrease of the Price Index are a mathematical consequence of a literature that is growing exponentially. Hence, he argues, these phenomena do not have any special scientometric reason (Egghe, 2010). 


\section{P.I. 10 years over time}

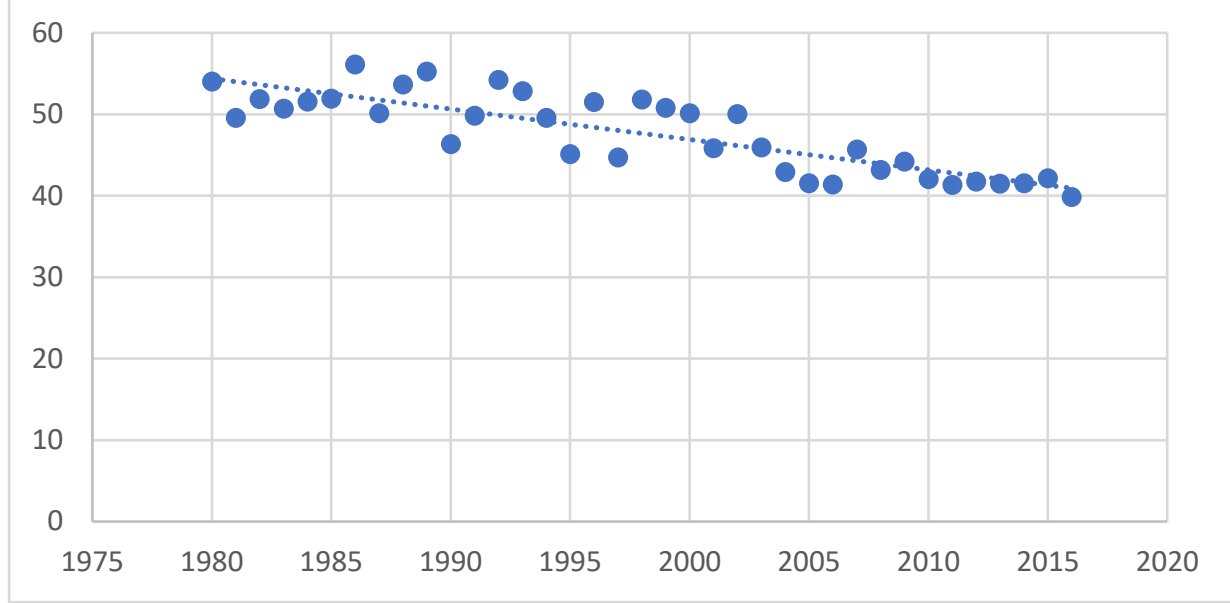

Figure 2. Price Index of Top Five Analytic Philosophy journals (10 years)

Considering these results and the related literature (in particular the study by Larivière et al.), I think that further research is needed to investigate the relationship between the aging of the literature and the transition to a normal science phase. In the present state of research, we lack a clear epistemological interpretation of the process of literature aging. I believe that this is a topic worth investigating, especially by scientometrics-minded philosophers of science, and I hope that in the future more studies will deal with it.

\section{Cited references}

Egghe, L. (2010). A model showing the increase in time of the average and median reference age and the decrease in time of the Price Index. Scientometrics, 82(2), 243-248. https://doi.org/10.1007/s11192-0090057-3

Glänzel, W., \& Schoepflin, U. (1995). A bibliometric ageing study based on serial and non-serial reference literature in the sciences. In Proceedings of the fifth biennial conference of the ISSI (pp. 177-185). River Forest, IL: Rosary College.

Larivière, V., Archambault, É., \& Gingras, Y. (2008). Long-term variations in the aging of scientific literature: From exponential growth to steady-state science (1900-2004). Journal of the American Society for Information Science and Technology, 59(2), 288-296. https://doi.org/10.1002/asi.20744

Petrovich, E. (2018). Accumulation of knowledge in para-scientific areas: the case of analytic philosophy. Scientometrics. https://doi.org/10.1007/s11192-018-2796-5 
Price, D. J. de S. (1986). Citation measures of hard science, soft science, technology, and nonscience. In Communication among scientists and engineers (pp. 155-179). New York: Columbia University Press.

Rescher, N. (2014). Metaphilosophy: philosophy in philosophical perspective. Lanham: Lexington Books.

Wray, K. B. (2016). No new evidence for a citation benefit for Author-Pay Open Access Publications in the social sciences and humanities. Scientometrics, 106(3), 1031-1035. https://doi.org/10.1007/s11192-016$1833-5$ 